\title{
Influence of fracture incubation time on dynamic crack propagation in brittle solids
}

\author{
Aleksandr Grigoriev ${ }^{1, *}$, and Evgeny Shilko ${ }^{1,2}$ \\ ${ }^{1}$ Institute of Strength Physics and Materials Science, SB RAS, 2/4 pr. Akademicheskii, 634055, \\ Tomsk, Russia \\ ${ }^{2}$ National Research Tomsk State University, 36 Lenina ave., 634050, Tomsk, Russia
}

\begin{abstract}
The paper is devoted to theoretical study of the longitudinal shear (mode II) crack unstable growth dynamics in brittle materials. We considered two main regimes of the dynamic propagation of the crack (sub-Rayleigh and supershear) and their implementation conditions. The research was carried out by computer simulation with the Movable Cellular Automaton method, using the generalized kinetic fracture model, which takes into account the finite duration of local fracture (fracture incubation time). It is shown that the fracture incubation time is a key parameter, which determines the transition conditions of the shear crack growth process from the sub-Rayleigh regime to supershear.
\end{abstract}

\section{Introduction}

One of the topical issues of the modern fracture mechanics is determination of regularities and identification of conditions for the realization of possible dynamic propagation regimes for longitudinal shear crack in brittle materials [1-3]. The topicality of the problem solution is determined by the importance of reliable prediction of the intensity and elastic wave radiation spectrum by a growing crack, including evaluation of the Earth's crust fault zones seismic potential. The regime of faulting determines the duration and the amplitude of generated longitudinal and transverse elastic oscillations as well as the ratio between $\mathrm{P}$ and $\mathrm{S}$ wave energies (that is, seismic manifestations of earthquakes) [4-6].

Within the framework of the classical linear elastic fracture mechanics, the unstable shear crack growth rate in a brittle material is determined by the equivalent stress value at the crack tip and is limited above by the Rayleigh wave speed value $V_{R}$. However, many theoretical and experimental studies show that the shear crack velocity can exceed the $S$ wave speed and reach the P-wave speed in the material (the supershear regime) [7-9]. The sub-Rayleigh regime is well described using the linear fracture theory formalism, while the supersonic regime description requires the use of more complex models (cohesive zone models) $[4,10,11]$. In the framework of such models, it is assumed, as a rule, that the time duration of the local fracture process is negligible. This simplification is not fundamental while we consider slow processes; however, it begins to have a significant impact on the modeling results at process rates comparable to the elastic wave velocities in the material.

* Corresponding author: grigoriev@ispms.ru 
In fact, the shear crack growth dynamics is largely determined by the rate of local fracture incubation processes near the crack tip. Therefore, when modeling the crack propagation processes in the supershear regime, it is important to take into account the finite value of fracture incubation time. The fracture incubation time can be taken into account by using fracture models based on the concepts of the kinetic theory of strength [12-14]. One of such models was recently proposed by the authors of the presented work [15]. Let us note that the fracture incubation time parameter integrally characterizes intensity of the process of discontinuities growth and coalescence at lower (in comparison with the considered) spatial scales.

The objective of the paper is to study the effect of the local fracture incubation time value on the shear (mode II) crack growth dynamics in brittle solids and on implementation conditions for the supershear regime of crack propagation. The research was carried out by computer simulation with the use of the movable cellular automaton (MCA) method and the fracture model, proposed by the authors and based on the concepts of the kinetic theory of strength [15].

\section{Model description}

The MCA method belongs to the class of "explicit" discrete elements methods, which use the principle of "local" force balance $[16,17]$. The key advantage of the MCA method in comparison with the traditional implementations of the "explicit" discrete elements methods is the use of a many-body formulation of the relations for element-element interaction forces [17]. The consolidated material fragment is modeled by an ensemble of linked (chemically bound) pairs of elements. Such pairs resist compression, tension and shear. Unbound pairs can show only contact interaction. Local fracture is modeled by "switching" the type of the pair interaction from bound to unbound [16, 18]. The switching is criterial. Both "force", and strain and energy-based criteria of local fracture can be applied. This paper uses, proposed by the authors, generalized dynamic formulation of the Drucker-Prager criterion, which takes into account the finite duration of local fracture in the material [15]:

$$
\sigma(t)=\sigma_{D P}(t)=\sigma_{e q}(t) 0.5(a+1)+\sigma_{\text {mean }}(t) 1.5(a-1) \geq \sigma_{c}^{d y n}\left(t-t_{0}\right),
$$

where $a=\sigma_{c}^{d y n} / \sigma_{t}^{d y n}, \sigma_{c}^{d y n}=\sigma_{c}^{d y n}\left(T_{f}\right)$ is the dynamic compressive strength, $\sigma_{t}^{d y n}=\sigma_{t}^{d y n}\left(T_{f}\right)$ is the dynamic tensile strength, $T_{f}=t_{f r}-t_{0}$ is the fracture incubation time, $t=t_{f r}$ is the fracture moment. Let us note that criterion (1) is constructed within the framework of the brittle materials dynamic behavior model, based on the principles of the structural-kinetic theory of strength and developed by the authors [15]. To apply the criterion (1) for the considered material, it is necessary to have "calibration" dependences of the dynamic strength values on the fracture time. In this paper, we used the dependencies previously proposed by the authors to model the dynamic loading of elastic-brittle material macroscopic samples [15].

We modeled the process of longitudinal shear crack propagation in a $2 \mathrm{D}$ sample, which consists of two finite thickness plates with the same mechanical properties connected by an interface (Fig. 1). The problem was considered in the approximation of a plane strain state. A short initial crack was assigned on the interface as a series of unbound discrete elements. In the presented calculations, the plates length was $8 \mathrm{~m}$, the height $-0.7 \mathrm{~m}$, and the discrete element diameter $D_{\text {elem }}=1 \mathrm{~cm}$. 


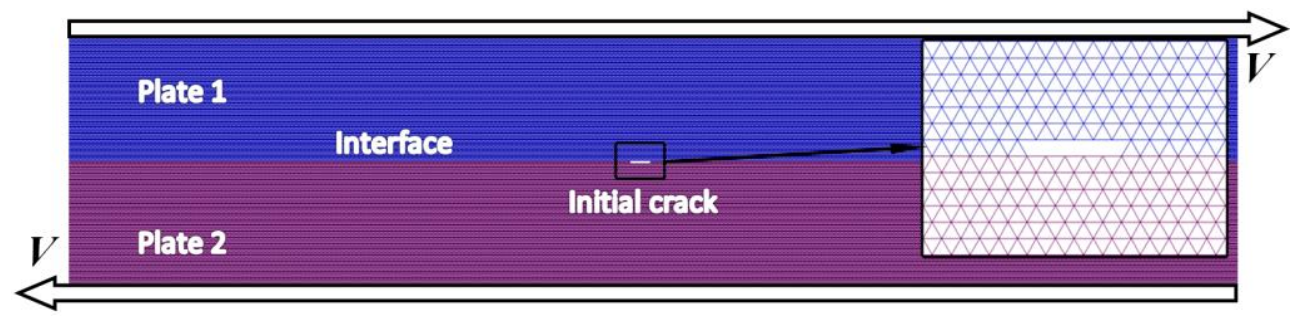

Fig. 1. Structure of the sample and the loading scheme.

The sample was deformed by the upper and lower plates surfaces tangential displacement in opposite directions at low velocity (in the quasistatic regime). The vertical positions of these surfaces were fixed, what ensured the simple shear condition fulfillment. In the horizontal direction, periodic boundary conditions were applied to the sample. Let us note that such a statement of the problem (with the "weakened" interface) is typical for modeling longitudinal shear crack due to shear crack tendency of trajectory distortion.

The plates material was assumed to be linear-elastic and high-strength (indestructible). The elastic characteristics and the density of the model brittle material corresponded to characteristic values for consolidated sandstones: density $\rho=2200 \mathrm{~kg} / \mathrm{m}^{3}$, Young's modulus $E=20 \mathrm{GPa}$, Poisson's ratio $v=0.2$. The interface, connecting the plates, had the strength which is characteristic for consolidated sandstones: $\sigma_{\mathrm{c}}{ }^{s t}=70 \mathrm{MPa}, \sigma_{t}{ }^{s t}=23.3 \mathrm{MPa}(a=3)$.

\section{Results and discussion}

The process of the sample loading is accompanied by elastic strain energy accumulation in its volume. Herewith, the maximum shear stresses are concentrated at the initial crack tips. When the applied shear stress reaches a limiting value, which depends on the initial crack geometric characteristics and is a certain fraction of the interface shear strength, the crack begins to grow in the dynamic regime. We consider the motion of one of the crack tips.

Figure 2 shows an example of the classical dependence of the velocity of dynamic crack tip propagation in the sub-Rayleigh regime on the distance made by the crack tip. This dependence was obtained in the approximation of an infinitely small fracture incubation time (the approximation used in the classical models of fracture mechanics). The Figure shows two main stages of the crack growth process. At the first stage, the crack tip propagation velocity increases and reaches a standing (saturation) value. The second stage of the crack growth process is characterized by a constant crack tip propagation velocity. In this case, the average crack tip propagation velocity value in the steady state phase of propagation is close to the Rayleigh wave speed in the material. This corresponds to the analytical estimates and the modeling results of other authors for the "sub-Rayleigh" regime of the dynamic crack propagation. 


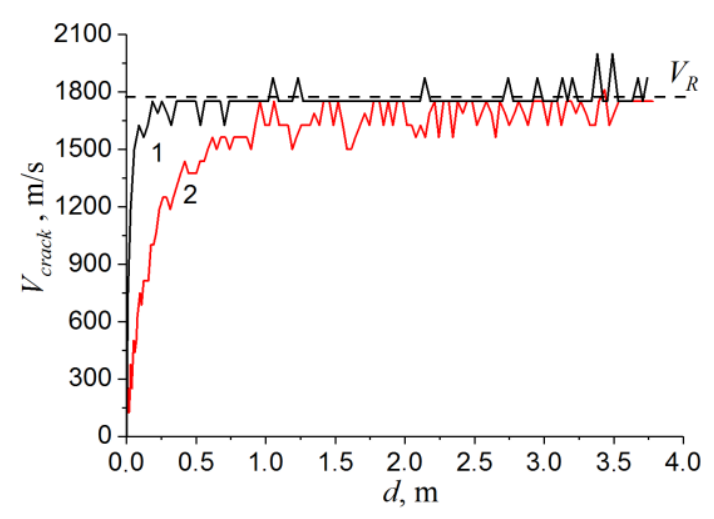

Fig. 2. The crack tip propagation velocity $V_{\text {crack }}$ dependences on the crack tip path length $d$ for the cases of infinitely small fracture incubation time (curve 1) and finite fracture incubation time (curve 2).

The finite fracture incubation time consideration leads to the fact that the dynamics of crack growth velocity at the initial phase of crack propagation in the sample slows down significantly (Fig. 2, curve 2). In particular, the path made by a crack tip before reaching the steady dynamic growth regime is approximately 4 times longer than in the case of "instantaneous" local fracture. In addition, the steady-state value of the crack velocity decreases by at least $5-6 \%$ and it better corresponds to the experimental data for low- or non-porous brittle materials (in most cases, the steady-state value of the longitudinal shear crack velocity in brittle materials does not exceed 0.8-0.9 Rayleigh wave speed).

In previous works of the authors $[19,20]$, with the use of one of the classical twoparameter fracture criteria (in approximation of the infinite small fracture incubation time), it was shown that an elastic vortex, which is a shear stress concentrator, is already formed in the initial phase of longitudinal shear crack propagation ahead of the crack tip. The elastic vortex moves ahead of the tip with the shear wave speed, and as it moves, the values of vorticity and shear stress concentration in the vortex gradually increase due to the influx of elastic strain energy from the unloading sample part behind the crack tip. Since the elastic vortex moves faster than the crack tip, after some time it moves away from it (herewith the vortex loses its "energy supply") and it becomes an independent dynamic object. The paper [19] is the first to show that the elastic vortex is a physical mechanism for accelerating cracks to "supershear" velocities: if the stress value in an elastic vortex has time to reach the critical value (material strength) before the elastic vortex separates from the crack, it leads to formation of a short "daughter" shear crack in its central part, which propagates in the supershear regime.

The power of energy inflow into the elastic vortex from the unloading material areas behind the crack tip is mainly determined by the elastic strain energy density accumulated in the material by the time the crack growth starts. It is effectively characterized by the shear strength of the material with the initial crack $\tau_{s t}$. In the paper [19], it was shown that $\tau_{s t}$ is a single-valued function of the initial crack dimensionless geometric parameter $P=L / W$ ( $L$ is the initial crack length, $W$ is its effective width) The corresponding relation, generalizing the classical Griffiths formula was empirically obtained. On this basis, there was proposed a geometrical criterion, determining the potential ability of the initial straight crack in the material to accelerate to supershear rates: at $P<P_{\text {crit }}$ the crack can become supershear, at $P>P_{\text {crit }}$ it can propagate only in the classical "sub-Rayleigh" regime. The threshold value $P_{\text {crit }}$ is a material parameter (for elastic-brittle materials it varies in the characteristic range from several units to 10-15) and is expressed by the difference between the longitudinal and Rayleigh elastic waves speeds and the material viscosity [20]. 
The threshold $P_{\text {crit }}$ is determined by the crack acceleration dynamics at the initial phase of its growth (before the elastic vortex separation). Therefore, the value of $P_{\text {crit }}$ should obviously increase when taking into account the finite duration of local fracture because fracture incubation time affects the crack growth dynamics at the initial phase (Fig. 2, curve 2 ). To verify this assumption we performed a series of the sample shear deformation simulations with initial cracks characterized by different $P$ parameter values. We used two described above approximations: instantaneous fracture (the classical criterion formulation) and finite fracture time (the dynamic formulation (1)).

Figure 3a shows the simulation results of the crack tip propagation velocity dynamics for the initial cracks, which are characterized by different $P$ parameter values, with the use of instantaneous local fracture approximation. It can be seen that when the initial crack parameter exceeds the threshold value (in this case $P_{\text {crit }}^{\text {st }} \approx 4.6$ ), the crack can propagate only in the classical sub-Rayleigh regime.

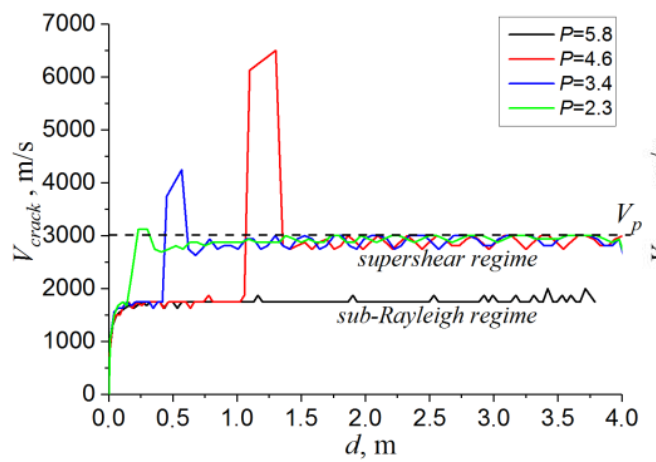

a)

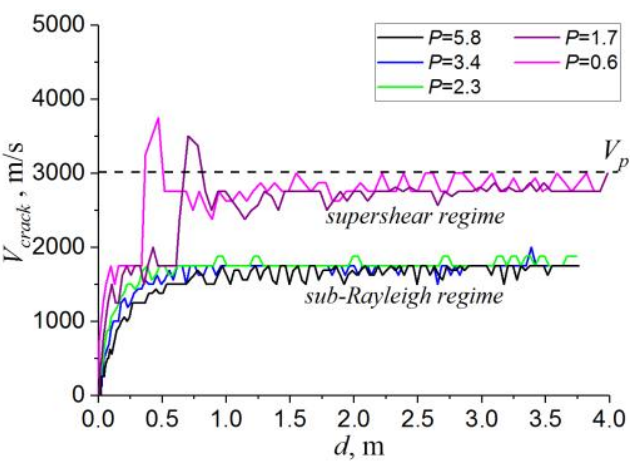

b)

Fig. 3. The crack tip propagation velocity $V_{\text {crack }}$ dependences on the crack tip path length $d$ for samples with different initial crack lengths: a) infinitely small fracture incubation time; b) finite fracture incubation time (criterion (1)).

Figure $3 b$ shows similar calculated curves, obtained with the use of the fracture model (1) with finite value of fracture incubation time. It can be seen that the maximum length of the initial phase, during which a daughter crack is supposed to appear in the elastic vortex, is approximately the same for the dynamic and "quasistatic" fracture models. This is logical as the elastic vortex is an independent dynamic object, and the crack growth dynamics can affect only the energy inflow power into the elastic vortex, but not its movement. At the same time, introduction of the finite time of local fracture leads to the $P_{c r i t}$ value decrease by approximately 3 times in comparison with the "instantaneous" approximation. Let us note that special calculations showed that the functional form of the $P_{c r i t}$ value dependence on the material parameters (elastic moduli, viscosity) [20] is preserved.

A detailed analysis of the $P_{\text {crit }}$ dependence form on the local fracture incubation time is the goal of further research.

\section{Conclusion}

The paper reviewed the numerical study of the longitudinal shear crack unstable growth process peculiarities in brittle solids. It was shown that the local fracture incubation time value (the time of preparatory processes in the cohesive zone ahead of the crack tip) is a material parameter, which determines not only the crack growth dynamics at the phase of reaching the steady state propagation, but also the condition for its transition to the supershear regime of growth (propagation with the speed exceeding the transverse elastic 
wave velocity in the material). Introduction of the real (though small) time of relaxation of local stresses in brittle materials makes it possible to predict significantly more accurately the possible regimes and features of crack propagation dynamics under shear conditions, and consequently, the elastic waves radiation peculiarities. The latter is especially important for an adequate assessment of seismic potential the fault zones high-stress fragments.

The work was carried out in the framework of the Fundamental Research Program of the State Academies of Sciences for 2013-2020 (Priority direction III.23).

\section{References}

1. F. Barras, R. Carpaij, P.H. Geubell, J.-F. Molinari, Phys. Rev. E 98, 063002 (2018)

2. D.S. Kammer, I. Svetlizky, G. Cohen, J. Fineberg, Sci. Adv. 4, eaat5622 (2018)

3. M. Mello, H.S. Bhat, A.J. Rosakis, J. Mech. Phys. Solids 93, 153 (2016)

4. E.M. Dunham, R.J. Archuleta, Geophys. Res. Letters. 32, L03302 (2005)

5. B.T. Aagaard, T.H. Heaton, Bull. Seism. Soc. Am. 94, 2064 (2004).

6. H.S. Bhat, R. Dmowska, G.C.P. King, Y. Klinger, J.R. Rice, J. Geophys. Res., B, Solid Earth 112, 1 (2007).

7. F.F. Abraham, H. Gao, Phys. Rev. Lett. 84, 3113 (2000).

8. J.-P. Ampuero, Y. Ben-Zion, Geophys. J. Int., 173, 674 (2008).

9. M. Bouchon, M. Vallee, Science 301, 824 (2003).

10. K.B. Broberg, Geophys. J. Int. 119, 706 (1994).

11. B.L. Karihaloo and Q.Z. Xiao, Phys. Mesomech. 22(1), 18 (2019)

12. N.F. Morozov, Yu.V. Petrov, Eur. J. Mech. A/Solids 25(4), 670 (2006).

13. Yu.V. Petrov, B.L. Karihaloo, V.V. Bratov, A.M. Bragov, Int. J. Eng. Sci. 61, 3 (2012).

14. Yu.V. Petrov, Soviet Physics Doklady 36, 802 (1991).

15. A.S. Grigoriev, E.V. Shilko, V.A. Skripnyak, S.G. Psakhie, Int. J. Imp. Eng. 123, 14 (2019).

16. D.O. Potyondy, P.A. Cundall, Int. J. Rock Mech. Min. Sci. 41 (8), 1329 (2004).

17. E.V. Shilko, S.G. Psakhie, S. Schmauder, V.L. Popov, S.V. Astafurov, A.Yu. Smolin, Comp. Mater. Sci. 102, 267 (2015).

18. S. Wu, X. Xu, Rock Mech. Rock Eng. 29, 1813 (2016)

19. S.G. Psakhie, E.V. Shilko, M.V. Popov, V.L. Popov, Phys. Rev. E 91, 063302 (2015).

20. E.V. Shilko, S.G. Psakhie, V.L. Popov, AIP Conf. Proc. 1683, 020209 (2015). 